\title{
EMPLEO DE ESPIRONOLACTONA-ISOBUTILHIDROCLOROTIAZIDA EN TOXEMIA GRAVIDICA Y ENFERMEDADES AFINES
}

TRABAJO REALIZADO EN EL "SERVICIO DE TOXEMIAS" DEL INSTITUTO MATERNO INFANTIL DE BOGOTA, COLOMBIA S. A.

\author{
Dr. Jesús Alberto Gómez Palacino(棌) \\ Dr. Jaime Rodríguez T.(棌)



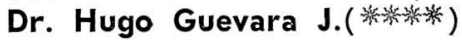

\section{Introducción e hipótesis}

Dentro de los fenómenos fisiopatológicos peculiares de la toxemia gravídica, el desequilibrio hidroelectrolítico juega un papel muy importante a tal punto que según Martin y Mills (24), la gestosis o disgravidia es debida, entre otras causas, a la pérdida del equilibrio entre las substancias sodioretenedoras y las sodioexcretoras incluyendo entre las primeras a las hormonas corticoadrenales, y particularmente a la Aldosterona, y entre las segundas a la progesterona.

A pesar de las múltiples investigaciones con respecto al papel del sodio en la toxemia - cuya reabsorción patológica en los túbulos renales ha sido demostrada, entre otros autores por Chesley y cols. (7) - no se ha podido precisar con exactitud qué relación guarda con el vasoespasmo propio del síndrome. Finnerty (12) señala que la anormalidad en el metabolismo del sodio precede al vasoespasmo y que, por lo tanto, la corrección de la primera alteración debe evitar o disminuir las consecuer.cias de la segunda. Esta posibilidad ha sido con- firmada en los trabajos de De Alvarez (10) y Flowers y cols. (14).

Los efectos de la retención de sodio y agua pueden sintetizarse indicando que con ella aumenta el líquido intra y extracelular, favorece la disrritmia cerebral que precede a las convulsiones, aumenta la isquemia renal y el espasmo vascular, eleva la tensión arterial, incrementa la producción de hormona antidiurética, desencadena la proteinuria y disminuye el volumen plasmático total. Este último fenómeno, la hemoconcentración, es temible por su consecuencias: anoxia y anoxemia de los órganos vitales, acidosis, hiperpirexia y aumento de la disrritmia cerebral. La retención hidrosalina favorece la oliguriaanuria y ha sido además impugnada

(*) Profesor Titular de Obstetricia y Ginecología de la Facultad de Medicina Universidad Nacional. Profesor Facultad de Medicina Universidad del Rosario.

(**) Jefe del Laboratorio Clínico, Hospital San Juan de Dios. Bogotá.

$(* * *)$ Jefe de la Sección de Química del Laboratorio Clínico. Hospital San Juan de Dios. Bogotá.

$(* * * *)$ Especialista del Servicio de Toxemias. 
del desplazamiento extracelular del calcio con la consiguiente tetania, irritabilidad neuromuscular y aparición de convulsiones.

La Aldosterona es el principal regulador de sal en el organismo; su secreción por parte de la corteza suprarenal depende de las concentraciones de sodio y potasio en el suero, del volumen del líquido extracelular, de la liberación de renina por el aparato yuxtaglomerular del riñón $y$, en una pequeñísima medida, de la secreción de ACTH por la antehipófisis. La hipovolemia parece ser el estímulo más importante para su secreción. Chesley (9) y Chesley y cols. (8) piensan que el sistema renina-angiotensina sea regulador hormonal importante de la secreción de Aldosterona y que el Aldosteronismo secundario de la gestación puede deberse a la alta concentración de angiotensina endógena.

Rauschkolb y Farrel (27) y Farrel y cols. (13) han demostrado que un extracto diencefálico dado por vía endovenosa a perros descerebrados, aumenta la eliminación de Aldosterona en la sangre de la vena adrenal y que en perros hipofisectomizados la Aldosterona alcanza sólo a un $66 \%$ de lo normal. De lo anterior deducen los autores mencionados que el hipotálamo juega un papel importante en el control de esta hormona. Farrel, citado por Guyton (15) afirma la presencia de un centro hipotalámico que regula la liberación de Aldosterona a expensas de una hormona glomerulotrófica.

La Aldosterona no solamente altera la permeabilidad de la membrana celular, y por ende la constitución bioquímica de los distintos compartimentos, sino que actuando sobre el túbulo contorneado distal favorece la reabsorción del sodio y por consiguiente de agua. Es éste uno de los mecanismos que explica la presencia de edema observado en la mayor parte de las pacientes con preeclampsiaeclampsia.

Las complicadas investigaciones de Kumar y cols. (18) acerca del contenido de sodio en los tejidos y su relación con la Aldosterona le permitieron afirmar que el sodio intracelular está disminuído en embarazadas normales y notablemente aumentado en la toxemia y que en las primeras la excreción de Aldosterona es generalmente alta mientras que en las pacientes con toxemia suele estar disminuída.

El papel que la Aldosterona desempeña en el complejo mecanismo fisiopatogénico de la toxemia gravídica ha sido explicado por Patterson (25) en la siguiente forma: al comienzo del desarrollo de la gestosis, cuando hay un grado moderado de degeneración de la placenta, aparece un exceso de angiotensina que estimula a la suprarenal a secretar en cantidad apreciable Aldosterona la cual produciría retención de sodio y líquidos en el espacio extravascular. La combinación del exceso de angiotensina y Aldosterona ocasionaría espasmo arteriolar e hipertensión arterial. La Aldosterona libre, con efecto específico sobre la membrana celular obligaría a las células a absorber los iones extras de sodio, que, junto con los contenidos en el espacio intersticial ocasionarían el edema periférico. Cuando la degeneración de la placenta progresa y la toxemia avanza, pequeñas cantidades de angiotensina pueden mantener elevada la tensión arterial. Finalmente el nivel de angiotensina y la resultante secreción de aldosterona pueden caer a niveles muy bajos y a pesar de lo cual la toxemia continúa porque el disturbio del sodio probablemente persiste dada la gran cantidad del ion almace- 
nado en el fluído extra e intracelular. La lesión de los vasos renales progresará tanto como lo sea el espasmo arteriolar y la hipertensión, aunque los índices de angiotensina hayan descendido a niveles bajos.

El aumento de Aldosterona en la sangre de pacientes con toxemia ha sido confirmado entre otros investigadores por Jones y cols. (16) pero Erlich (11) afirma que tal aumento es inferior al que en embarazadas normales debe ocurrir y que si el efecto sodioretenedor es más manifiesto en las primeras ello se debe a deficiencia de progesterona.

La importancia de la Aldosterona en relación con la disgravidia también ha sido investigada por Kumar y col. (17) siguiendo 3 rutas: A - Por el estudio de los efectos sobre las ratas; B - Por determinación de la hormona en la orina de pacientes con toxemia, y C - For extracción de Aldosterona de la placenta tratando de buscar una posible fuente adicional de la hormona. Los niveles bajos de Aldosterona hallados por los citados autores en la orina de pacientes con toxemia en relación con pacientes normales, les permite sugerir la posibilidad de que el fenómeno puede deberse a cambios metabólicos en riñón e hígado 0 a disminución de la rata de filtración plasmática por parte del glomérulo.

Los estudios de Rinsler y Risby (29) y Martin y Mills (24), demostraron disminución en la eliminación urinaria de Aldosterona en pacientes con toxemia al paso que Leutscher $y$ Curtis (20), Prunty (26) y Barnes y Quilligan (3) la encontraron aumentada. No obstante, como lo afirma Rodríguez-Argüelles (31) debe advertirse que la cantidad de hormona excretada puede guardar muy poca relación con los niveles sanguíneos de Aldos- terona o con la cantidad que se produce y se utiliza en el organismo.

Perturbada la distribución iónica en la toxemia gravídica, uno de los objetivos fundamentales de su terapéutica es restablecer el equilibrio hidroelectrolítico y la función excretora del riñón. Con tal fin se han utilizado diferentes medidas y fármacos de efecto diurético y natriurético.

La retención sódica ocasionada por la Aldosterona puede ser controlada según Richter y Arnold (28): A - corrigiendo la causa del aldosteronismo, $B$ - inhibiendo los mecanismos renales de reabsorción de sodio por medio de fármacos, C - inhibiendo la sintesis de Aldosterona con Metopirona, y D - antagonizando la acción de la Aldosterona sobre los túbulos renales.

Un avance importante de la farmacología moderna ha sido el descubrimiento de sustancias con propiedades antihormonales, dentro de las cuales se incluye la espironolactona, cuyos efectos bloqueadores sobre la Aldosterona favorecen el restablecimiento de la homeostasis y el medio iónico perdidos.

La espironolactona, o Aldactona, es la lactona del ácido 3-(3-oxo 7 alfa acetiltio-17 $\beta$ hidroxi-4-androeten - 17 propiónico. Producto sintético, pertenece al grupo de los compuestos químicos llamados esteroides.

La espironolactona bloquea específicamente la acción retentiva del sodio y excretora del potasio que tiene la Aldosterona sobre el túbulo distal.

La espironolactona sola ha sido utilizada en el control de la toxemia gravídica. La bondad de sus efectos ha sido demostrada por Arnold $y$ Richter (1) quienes encontraron: 1? 
que la excreción urinaria de electrolitos en la toxemia está disminuída, 2 . que la aldactona no tiene efectos en pacientes embarazadas normales, 3 : que la excreción de sodio, cloro, potasio y agua aumentan con la administración del fármaco en pacientes con gestosis, 4: que sus efectos se manifiestan desde el segundo día de su prescripción y $5^{\circ}$ que el peso de las enfermas y la excreción de proteínas no sufrieron modificaciones sensibles aunque sugirieron que tales parámetros podrían alterarse usando closis más altas del medicamento 0 por más tiempo.

Maroudis y cols. (22) demostraron que $400 \mathrm{mg}$. de Aldactone diariamente administrados durante 8 días a 24 pacientes con toxemia produjeron: A - baja de la presión arterial, B - desaparición del edema, C - disminución de la proteinuria y D - disminución del sodio hemático.

Magliavacca y Bompiani (21) estucliaron el efecto de la espironolactona en 6 pacientes con síndrome toxémico administrando de 400 a 800 mg. por día habiendo observado aumento de la diuresis, de la eliminación de sodio y cloro sin compromiso mayor para el potasio, y disminución de la tensión arterial. Advierten que tales efectos no fueron igualmente satisfactorios en todas las enfermas.

Las experiencias anteriores no son concordantes con las de Buckingham y Barnes (5) quienes afirman que la espironolactona produjo en sus estudios marcada eliminación de sodio en embarazadas normales a término y no ocasionó efecto en pacientes preeclámpticas.

Con base en las apreciaciones anteriores, y dadas las discrepancias vigentes, nos decidimos a utilizar la espironolactona en el manejo de la to- xemia gravídica y enfermedades afines. Con tal objeto, y siguiendo las recomendaciones de Magliavacca y col. (Op. cit.) y de Massey (23) utilizamos la asociación de la sustancia en mención junto con la isobutilhidroclorotiazida, derivado éste último cuya acción terapéutica ha sido considerada 10 veces superior a la de su predecesor la hidroclorotiazida.

Las tiazidas, sustancias derivadas de las sulfonamidas, poseen un efecto diurético marcado con la consiguiente eliminación no sólo de sodio sino también de potasio. Se ha discutido mucho el sitio y mecanismo de su acción. Laroche y Coquet (19) afirman que actúan a nivel del túbulo proximal deprimiendo la reabsorción de sodio y cloro. Wilson (33) y Flowers (14) concluyen que su acción se realiza especialmente en el túbulo distal; lo más probable es que impidan la reabsorción del sodio a diversos niveles del nefrón. Crawford y col. (6) supone que actúan inhibiendo un sistema enzimático semejante a la anhidrasa carbónica, sistema éste aún no bien conocido.

Las tiazidas aumentan la eliminación de potasio lo cual ha sido relacionado con su efecto inhibidor de la anhidrasa carbónica y que se explica porque al disminuir la reabsorción del sodio en el túbulo proximal, en donde tiene lugar el intercambio de sodio por potasio, aumenta la eliminación de éste.

Las tiazidas según diversos autores $(2,4,30)$ tienen un efecto hipotensor que depende de la saluresis que provocan pero además existe cierto factor en su estructura molecular con alguna capacidad para inhibir la resistencia periférica.

La posibilidad de utilizar preparaciones con diversa acción farmacodi- 
námica, y en nuestra experiencia la asaciación espironolactona - isobutilhidrociorotiazida, brinda en la actualidad la oportunidad de potencializar sus efectos favorables disminuyendo los indeseables.

\section{Material y Métodos}

La asociación espironolactona - isobutilhidroclorotiazida* fue administrada a pacientes con los siguientes diagnósticos:

\section{Preeclampsia.}

\section{Eclampsia.}

Enfermedad vascular crónica hipertensiva - EVCH- y toxemia sobreagregada.

Enfermedad vascular crónica hipertensiva sin toxemia.

Cardiopatía reumática con hipertensión arterial.

\section{Síndrome nefrótico.}

El diagnóstico diferencial de las distintas entidades se estableció con base en la anamnesis, la época de aparición de signos y síntomas y por los procedimientos clínicos y de laboratorio habituales.

Como pacientes con preeclampsia se clasificaron aquellas enfermas cuya signología apareció después de la semana 24 de la gestación, ordinariamente primigestantes, y sin patología renal o hipertensiva previas a la gestación.

Como pacientes con EVCH fueron incluídas aquellas enfermas cuyo signo fundamental fue el aumento de la presión arterial —diastólica por encima de $90 \mathrm{~mm}$. de $\mathrm{Hg}$ - puesto de manifiesto antes del embarazo o de la semana 24. Cuando al fenómeno anterior se adicionó el aumento de la tensión sistólica en $30 \mathrm{~mm}$. o más o a la diastólica de $15 \mathrm{~mm}$. o más, o cuando apareció proteinuria súbita después de la semana 24 , se clasificaron las pacientes con EVCH con toxemia sobreagregada.

El diagnóstico de cardiopatía reumática con hipertensión y de síndrome nefrótico se orientó de acuerdo con los procedimientos usuales.

Previamente a la iniciación de la terapia las pacientes fueron valoradas integralmente tanto en el aspecto médico general como en el obstétrico. Fueron sometidas a un período de observación de 24 horas durante las cuales se registraron los datos de peso, tensión arterial, edemas, pulso y diuresis a más de las condiciones propias del embarazo: edad gestacional en semanas, vitalidad fetal, etc.

Los exámenes de laboratorio previos a la terapia fueron:

Parcial de orina

Cuadro hemático

Rh y grupo sanguíneo

Creatinemia, azoemia, uricemia

Glicemia

Serología

lonograma ( $\mathrm{Na}, \mathrm{Cl}, \mathrm{K}$ ) en sangre $\mathrm{y}$ en orina.

El régimen dietético proporcionado a las pacientes en estudio no tuvo limitaciones ni en la ingestión de líquidos ni en la de sodio habiendo recibido de éste último un promedio de 10 gramos por día. Las pacientes deambularon a voluntad dentro del servicio, y no recibieron - salvo por circunstancias ocasionales y de excepción- atro tipo de medicamentos.

\footnotetext{
* ALDAZIDA, de Searle, Inter. American Co.
} 
El control clínico se anotó diariamente y los exámenes de laboratorio: ionograma en sangre y orina, azoemia y glicemia se repitieron los días $3^{\circ}, 6^{\circ}$ y $10^{\circ}$ del tratamiento. El manejo obstétrico siguió los lineamientos y pautas establecidas en el Servicio.

Se estudiaron 30 pacientes: 11 tenían preeclampsia, 9 eclampsia, 4 $\mathrm{EXCH}+$ toxemia, $4 \mathrm{EVCH}, 1$ cardiopatía reumática con hipertensión y 1 síndrome nefrótico.

La dosis diaria de la asociación medicamentosa fue de $100 \mathrm{mg}$. de espironolactona y 10 de isobutilhidroclorotiazida ( 4 comprimidos al día) y el tiempo de experiencia de 10 días.

La investigación se llevó a cabo en el Servicio de Toxemias del Instituto Materno Infantil de Bogotá, Colombia, S. A.

\section{Resultados}

Las efectos de la medicación fueron evaluados de acuerdo con los siguientes aspectos:

1. Disminución del peso de las enfermas
2. Disminución de la tensión arterial

3. Disminución de los edemas

4. Aumento de la diuresis

5. Aspectos bioquímicos

6. Resultados obstétrico - pediátricos.

\section{Preeclampsia}

No de casos: 11.

Los resultados correspondientes a los parámetros 1 a 4 fueron positivos. La disminución del peso osciló entre 1 kilo en dos casos hasta 7 kilos en uno, siendo el promedio de $3.1 \mathrm{~kg}$. en 10 días. La presión arterial descendió en todos los casos habiéndose logrado cifras normales en 3 enfermas. La disminución de los edemas fue total en 6 pacientes y en el resto el descenso fue apreciable. El aumento de la diuresis se logró en todas las enfermas y en una de ellas se lograron $3.445 \mathrm{cc}$. de orina en 24 horas; en una paciente la diuresis se duplicó al 2 : día de tratamiento. El mayor efecto excretor se observó entre el $5{ }^{\circ}$ y el 6: día en el grupo total de enfermas.

IONOGRAMA (CIFRAS PROMEDIO OBTENIDAS EN LAS 11 PACIENTES)

\begin{tabular}{rcccccc}
\hline & \multicolumn{2}{c}{ SODIO } & \multicolumn{2}{c}{ CLORO } & \multicolumn{2}{c}{ POTASIO } \\
Día & Sangre & Orina & Sangre & Orina & Sangre & Orina \\
\hline 19 & 139 & 118 & 103 & 96 & 4.8 & 48 \\
$3^{\circ}$ & 139 & 155 & 93 & 135 & 4.9 & 63 \\
$6^{\circ}$ & 121 & 178 & 103 & 135 & 5.0 & 61 \\
$10^{9}$ & 118 & 180 & 108 & 109 & 4.9 & 60 \\
\hline
\end{tabular}

El día $1^{\circ}$ corresponde al período de 24 horas de observación sin tratamiento.

Adviértase cómo la concentración de sodio plasmático estuvo inicialmente dentro de límites normales confirmándose así que su retención patológica en los procesos de toxemia gravídica tiene lugar en el compartimento intracelular e intersticial. Obsérvese cómo la tasa de sodio plasmático fue decayendo a medida que se avanzaba en el tratamiento. Los nive- 
les plasmáticos de cloro estuvieron dentro de límites normales y tanto éste como el sodio fueron aumentando su excreción urinaria reafirmándose de tal modo la acción natriurética de los fármacos. A pesar de que la excreción urinaria de potasio se vio algo aumentada, sus niveles plasmáticos se conservaron.

\begin{tabular}{rccc}
\hline Día & Azoemia & Uricemia & Glicemia \\
\hline $1^{\circ}$ & 16 & 8.7 & 102 \\
$3^{\circ}$ & 17 & 8.8 & 96 \\
$6^{\circ}$ & 18 & 9.3 & 94 \\
$10^{\circ}$ & 22 & 10.4 & 99 \\
\hline
\end{tabular}

Se observó aumento progresivo de los niveles de nitrógeno ureico habiéndose sobrepasado discretamente la cifra normal al término del tratamiento. La uricemia alta, propia de la toxemia, se vio ascender y las cifras de glucosa en sangre permanecieron normales.

\section{Resultados Obstétrico-Pediátricos}

6 fetos sobrepasaron los 2.500 grs. y 5 fueron prematuros. Hubo 3 mortinatos uno de los cuales pesó 1.000 grs. cuya madre tenía reacciones serológicas positivas. Una muerte fetal ocurrió intraparto y otro feto nació macerado con peso de 1.850 grs.

Se practicaron 2 cesáreas, una por distocia cervical y otra por presentación de pelvis en primigestante con ruptura prematura de membranas. 8 fetos sobrevivieron.

Todos los partos se iniciaron espontáneamente.

\section{Eclampsia}

№ de casos: 9.

Durante la fase convulsiva las enfermas fueron sometidas a tratamien- to según la metodología propuesta por Zuspan (34) a base de sulfato de magnesio en dosis inicial de 6 grs. por vía endovenosa administrados en 10 minutos y seguidos de una venoclisis de $1.000 \mathrm{cc}$. de Solución de Dextrosa al $5 \%$ en agua destilada con 24 gr. de Sulfato de Magnesio adicionales para ser perfundidos durante 24 horas intravenosamente.

Controlada la fase convulsiva y una vez entradas las pacientes en el llamado por Sánchez y cols. (33) Síndrome gravídico post-eclámptico, recibieron la combinación farmacológica en investigación.

La disminución del peso de las enfermas se observó en 7 de ellas y en 2 permaneció invariable. Llamó la atención el descenso de $18.5 \mathrm{k}$. en una paciente en el transcurso del tratamiento.

La presión arterial descendió en 6 enfermas, se mantuvo sin variación en 2 y en 1 caso ascendió. Se lograron niveles normales de tensión arterial en una paciente.

La disminución de los edemas se obtuvo en todos los casos y el aumento de la diuresis se logró en 8 de ellos habiéndose notado su mejor efecto diurético hacia el $4^{\circ}$ día de la terapia. Una paciente permaneció en oliguria durante todo el período de observación habiendo dado a luz un feto macerado.

El sodio y el cloro plasmáticos se mantuvieron dentro de límites normales, su excreción no se vio tan favorecida como en las pacientes con preeclampsia auncuando se notó el efecto salurético de los fármacos. No hubo modificaciones del potasio plasmático ni de su excreción urinaria. 
IONOGRAMA (CIFRAS PROMEDIO OBTENIDAS EN 9 PACIENTES)

\begin{tabular}{rrrrrrr}
\hline & \multicolumn{2}{c}{ SODIO } & \multicolumn{2}{c}{ CLORO } & \multicolumn{2}{c}{ POTASIO } \\
Día & Sangre & Orina & Sangre & Orina & Sangre & Orina \\
\hline $1^{\circ}$ & 138 & 126 & 103 & 130 & 5.0 & 65 \\
$3^{\circ}$ & 138 & 181 & 101 & 162 & 5.0 & 75 \\
$6^{\circ}$ & 120 & 146 & 99 & 120 & 5.0 & 56 \\
$10^{\circ}$ & 140 & 143 & 105 & 127 & 5.0 & 60 \\
\hline
\end{tabular}

El primer día corresponde al período de. 24 horas de observación sin medicación.

\begin{tabular}{cccc}
\hline Día & Azoemia & Uricemia & Glicemia \\
\hline $1^{\circ}$ & 18 & 9.9 & 107 \\
$3^{\circ}$ & 19 & 9.6 & 90 \\
$6^{\circ}$ & 25 & 10.5 & 102 \\
$10^{\circ}$ & 18 & 10.0 & 93 \\
\hline
\end{tabular}

No hubo aumento significativo del nitrógeno ureico. La hiperuricemia fue constante y aumentó con el transcurso de los días de tratamiento. El promedio de las cifras de glicemia en dos muestras fue ligeramente superior a 100 pese a que en el grupo había 2 pacientes diabéticas.

\section{Resultados Obstétrico-Pediátricos}

6 pacientes tuvieron hijos prematuros y 3 hijos fueron de término. Hubo 2 mortinatos, uno de 1.000 y otro de $2.000 \mathrm{gr}$. Un parto se indujo por reagravación de la sintomatología y 8

se desencadenaron espontáneamente. No se practicó ninguna cesárea.

\section{E.V.C.H. + Toxemia}

No de casos: 4.

La disminución del peso de las enfermas se obtuvo en 2 casos habiéndose logrado descenso de $12 \mathrm{~kg}$. en una paciente y de 8.5 en otra. La presión arterial se redujo en 3 casos sin lograr en ninguno de ellos niveles normales. En una enferma la tensión arterial era superior al terminar la terapia.

La reducción de los edemas se obtuvo en los dos casos en donde el signo estaba presente. La diuresis aumentó sensiblemente en un caso, en otro no fue importante $y$ en las pacientes que no tenían edemas la diuresis se mantuvo dentro de las cifras iniciales.

IONOGRAMA (CIFRAS PROMEDIO OBTENIDAS EN 4 PACIENTES)

\begin{tabular}{rrrrrrr}
\hline & & SODIO & & \multicolumn{2}{c}{ CLORO } & \multicolumn{2}{c}{ POTASIO } \\
Día & Sangre & Orina & Sangre & Orina & Sangre & Orina \\
\hline $1^{\circ}$ & 136 & 133 & 101 & 125 & 5.0 & 59 \\
$3^{\circ}$ & 137 & 320 & 102 & 283 & 5.0 & 111 \\
$6^{\circ}$ & 137 & 216 & 100 & 156 & 4.9 & 91 \\
$10^{\circ}$ & 139 & 150 & 108 & 115 & 5.0 & 67 \\
\hline
\end{tabular}

El día $1^{\circ}$ corresponde al período de 24 horas de observación sin tratamiento.

Pese a que los niveles plasmáticos de sodio y cloro estaban dentro de lo normal su excreción urinaria alcanzó niveles significativos, hasta de 320
$\mathrm{mEq}$ en 24 horas para el sodio y 283 para el cloro, habiéndose sobrepasado también la excreción de potasio en la segunda muestra. 


\begin{tabular}{cccc}
\hline Día & Azoemia & Uricemia & Glicemia \\
\hline 19 & 13 & 6.9 & 110 \\
$3^{\circ}$ & 14 & 8.3 & 94 \\
$6^{\circ}$ & 19 & 8.4 & 109 \\
$10^{\circ}$ & 18 & 9.2 & 70 \\
\hline
\end{tabular}

El nitrógeno ureico se mantuvo dentro de límites normales. La uricemia aumentó progresivamente bajo el tratamiento y la glicemia estuvo elevada en 2 pacientes una de las cuales era manifiestamente diabética.

\section{Resultados Obstétrico-Pediátricos}

Solamente un parto se efectuó a término y 3 fueron prematuros. Se practicó una cesárea por abruptio placentae y los otros partos se iniciaron y terminaron espontáneamente. Todos los fetos sobrevivieron.

\section{E. V. C. H.}

\section{Ni de casos: 4}

La pérdida de peso fue mínima en dos pacientes, 1 y 2 kilos, mientras que se mantuvo inmodificable en dos enfermas. La presión arterial tan solo descendió en un caso, en una paciente no se modificó y en dos hubo ascenso tensional.

Ninguna paciente mostraba edemas antes de la terapia y solamente hubo aumento de la diuresis en un caso auncuando no en cantidad significativa.

\section{IONOGRAMA (CIFRAS PROMEDIO OBTENIDAS EN 4 PACIENTES)}

\begin{tabular}{rcccccc}
\hline & & SODIO & \multicolumn{2}{c}{ CLORO } & \multicolumn{2}{c}{ POTASIO } \\
Día & Sangre & Orina & Sangre & Orina & Sangre & Orina \\
\hline 19 & 138 & 93 & 104 & 92 & 4.8 & 43 \\
$3^{9}$ & 131 & 157 & 111 & 113 & 4.9 & 62 \\
$6^{\circ}$ & 140 & 118 & 103 & 114 & 4.9 & 52 \\
$10^{\circ}$ & 138 & 158 & 105 & 123 & 5.0 & 57 \\
\hline
\end{tabular}

El día $1^{0}$ corresponde al período de 24 horas sin medicación.

Las cifras de sodio y cloro en sangre estaban dentro de lo normal y la excreción urinaria correspondiente fue notoriamente baja en relación con las pacientes de los grupos anteriores. El potasio en plasma era normal y su excreción en orina sufrió moderado aumento.

\begin{tabular}{rccc}
\hline Día & Azoemia & Uricemia & Glicemia \\
\hline & & & \\
$1^{\circ}$ & 10.7 & 5.6 & 93 \\
$3^{\circ}$ & 15.3 & 7.8 & 113 \\
$6^{\circ}$ & 16.5 & 9.7 & 126 \\
$10^{\circ}$ & 15.0 & 8.5 & 112 \\
\hline
\end{tabular}

El nitrógeno ureico permaneció normal, la uricemia se elevó discretamente y la glicemia se mostró aumentada siendo de destacar que en el grupo había una paciente diabética.

\section{Resultados Obstétrico-Pediátricos}

Tres enfermas tuvieron hijos prematuros y solamente una a término. Una paciente tenía embarazo gemelar. Hubo un feto mortinato, correspondiente a la paciente diabética. Se practicaron 2 cesáreas electivas extemporáneas, por empeoramiento de las condiciones clínicas, y las cuales fueron seguidas de esterilización quirúrgica. 


\section{CARDIOPATIA REUMATICA CON HIPERTENSION ARTERIAL}

Paciente de 17 años, $\mathrm{Gl}$, con estenosis mitral, insuficiencia tricuspídea e hipertensión arterial. Ingresó al Servicio con embarazo de 27 semanas. Fue sometida a la terapia en experimentación. El peso disminuyó $7 \mathrm{~kg}$. la presión sanguínea alcanzó niveles normales, los edemas desaparecieron y la diuresis aumentó sensiblemente.

Durante el tratamiento se observó que la excreción de sodio y cloro aumentó discretamente. La azoemia no experimentó cambios significativos. La uricemia moderadamente aumentada al comienzo se mantuvo así hasta el final de la terapia. La glicemia permaneció normal.

Nueve semanas más tarde la paciente dio a luz un feto vivo que pesó $2.250 \mathrm{gr}$.

\section{SINDROME NEFROTICO}

Paciente de 27 años, G3P2A0, con embarazo de 35 semanas y síndrome nefrótico. Fue tratada siguiendo el esquema terapéutico propuesto, durante 10 días. Las dosis de los medicamentos fueron luego disminuídas hasta el día en que se interrumpió el embarazo.

Durante la década de experiencia disminuyó el peso $7 \mathrm{~kg}$. los edemas disminuyeron de $11 \mathrm{I}$ a II grado, la proteinuria disminuyó de $3.15 \mathrm{gr}$. hasta $280 \mathrm{mg}$. y la diuresis aumentó hasta 3.445 cc. al $5^{\circ}$ día de tratamiento.

A las 39 semanas de gestación se practicó cesárea obteniéndose un feto vivo de $2.070 \mathrm{gr}$. seguida de esterilización quirúrgica.

Una biopsia renal efectuada en el puerperio informó Glomerulonefritis Membranosa y una cuantificación de proteínas en orina a los 45 días del parto informó $960 \mathrm{mg} . \%$.

\section{Resumen y Conclusiones}

Se hace una revisión de los factores involucrados en la retención hidrosalina como fenómeno fundamental dentro de la fisiopatogenia de la toxemia gravídica. Se destaca el papel que en tal desequilibrio desempeña la Aldosterona.

Se mencionan las principales experiencias realizadas por distintos investigadores acerca de la concentración plasmática de Aldosterona en pacientes embarazadas normales y toxémi- cas así como también las relaciones de la hormona con la distribución del sodio en los distintos compartimentos y su excreción urinaria.

Se recopilan algunos trabajos realizados utilizando la espironolactona como antihormona, útil en el manejo de la gestosis.

Se presentan los resultados clínicos, bioquímicos y obstétricos obtenidos con el empleo de la combinación de espironolactona - isobutilhidroclorotiazida.

El análisis estadístico demostró que la terapia en investigación utilizada en diversas formas y etapas de la toxemia y enfermedades afines produjo disminución del peso en 23 de 30 pacientes estudiadas, descenso de la presión arterial en 22, disminución de los edemas en 26 y aumento de la diuresis en 25. Estos efectos, altamente favorables, resultaron muy importantes si se tiene en cuenta que las pacientes no tuvieron limitación en el suministro ni de líquidos ni de sodio.

De 31 fetos, pues hubo un embarazo gemelar, 25 sobrevivieron y 6 fallecieron, estos últimos todos prematuros y de peso muy bajo. Se confirmó cómo en la toxemia gravídica los niveles plasmáticos de sodio y cloro estuvieron invariablemente normales, hecho importante si se reconoce que su retención patológica en los casos de disgravidia se confina al espacio intracelular e intersticial. Se observó cómo el efecto natriurético de la medicación fue evidente en todos los casos auncuando su mayor acción se manifestó en las enfermas con EVCH + toxemia y en las pacientes con preeclampsia, en contraste con los casos de EVCH sin toxemia en donde la excreción sódica fue mínima.

La pérdida de potasio por la orina fue despreciable en los casos de toxe- 
mia pura y apenas aumentó en las pacientes con patología subyacente a la toxemia.

Las cifras de nitrógeno ureico se vieron aumentar moderadamente a medida que transcurrió el tratamiento, fenómeno que interesó solamente a las pacientes con toxemia aguda mientras que en el resto de las enfermas la azoemia permaneció normal. Parecida conclusión se obtuvo con los niveles de ácido úrico, cuyo transporte parece estar perturbado a nivel tubular en los casos de toxemia, a diferencia de otros tipos de nefropatías.

La glicemia no sufrió modificaciones permaneciendo anormalmente alta en las pacientes diabéticas y normal en el resto de las enfermas. Los efectos colaterales 0 indeseables fueron muy reducidos anotándose tan sólo diaforesis en 3 casos, náuseas en 2, mareos y adinamia en 2 y rash pruriginoso en 1 caso.

Porque se lograron los objetivos buscados en el control de las manifestaciones clínicas de la toxemia gravídica y de las enfermedades afines, obteniéndose buenos resultados tanto en el aspecto médico como en el obstétrico, con el mínimo de efectos colaterales, consideramos que la asociación de la espironolactona-isobutil-hidroclorotiazida resulta ideal en el manejo de las gestosis.

La medicación demostró ser particularmente eficaz en el tratamiento del síndrome nefrótico durante la gestación.

\section{Summary and Conclusions}

A review was accomplished of the factors engaged in hydrosaline retention as a fundamental phenomeni within the physio-pathogeny of gravidic toxemia. The role played by aldosterone in such unbalance is outstanding.
The main experiences obtained by different investigators concerning the plasmatical concentration of aldosterone in normal and toxemical pregnant patients is mentioned, as well as the relationships of the hormone with the distribution of sodium in the different compartments and its urinary excretion.

A compilation is made of the works performed by means of expironolactone as an anti-hormone, useful in handling gestosis.

The clinical, biological and obstetrical results obtained with the use of the combination of hydrochlorotized isobutil-spirionolactone are shown.

The statistical analysis indicated that the therapy in the investigation used in the various stages of toxemia and similar illnesses produced a decrease in weight in 23 out of 30 patients studied, a decrease in blood pressure in 22, a decrease of edemas in 26 and an increase of diuresis in 25. These hyghly favorable effects are quite important when considering that the patients had no limits in the supply of either liquids or sodium.

Of 31 fetus, since there was one twin pregnancy, 25 survived and 6 died; the latter were all premature and with very low weight. It was confirmed that the plasmatical levels of sodium and chloride were invariably normal in gravidic toxemia, an important factor when accepting that their pathological retention in the cases of dysgravity is limited to the intracellular and interstitial space.

It was also observed how the natriuretical effect of the medicine was evident in all cases, although its greater effect was shown in patients with EVCH Toxemia and in patients suffering pre-eclampsia, in contrast with the cases of EVCH without toxemia 
where the sodium excretion was insignificant.

The loss of potassium through urine was un-important in the cases of pure toxemia and barely increased in patients with subjacent toxemia pathology.

The figures of ureical nitrogen slightly increased in the measure of development of the treatment, phenomena which only appeared in patients with acute toxemia, while azotemia continued normal in the rest of the patients. Like conclusiones were reached at the levels of uric acid, which conveyance seems to be disturbed at the tubular level in the case of toxemia, in comparison with other types of nephropathies.

Glycemia did not suffer modifications and remained abnormally high in diabetic patients and normal in the rest of the patients.

The collateral or unwanted effects were reduced; there were only 3 cases with diaphoresis, nausea in 2, seasickness and adynamia in 2 and a pruriginous rash in 1 case.

In view of having attained the objectives searched for control of clinical signs of gravidic toxemia and of similar illnesses, through good results in both the medical as the obstetrical aspects with a minimum of collateral effects, we believe that the association of hydrochlorotized isobutil-spirinoloactone is ideal for handling of gestosis. The medicine proved to be particularly efficient for treatment of the nephrotical syndrome during pregnancy.

\section{BIBLIOGRAFIA}

1 ARNOLD, M., y RITCHER, R.: Meeting of Schweiz, Ges. F. Gebh und Gyn., Interlaken, 15th June, 1961.
2 ASSALT, N. S., JUDD, L., MONDZN, S. N. y DASGUPTA, K.: J. Lab. and Clin. Med. 52: $423,1958$.

3 BARNES, A. C., Y QUILLIGAN, E. J.: Am. J. Obst. \& Gyn. 71: 670, 1956.

4 BOWER, D.: J. Obst. and Ginec. Brit. Comm 71 : 123, 1964.

5 BUCKINGHAM, J., y BARNES, A.: Surg. Forum. 9: 663, 1969.

6 CARWFORD, J. D., Y Mac GUILLIWRAY, M. H.: J. Pediat. 62: 413, 1963.

7 CHESLEY, L., VALENTI, C., Y REIN, H.: Metabolism. 7: 575, 1958.

8 CHESLEY, L. C. ; COSGROVE, R., y ANITTO, J.: J. Path. Microbiol.

9 CHESLEY, L. C.; Cls. Obs. y Gins. 878, 1966.

10 DE ALVAREZ, R. R.: Obst. and Gynec. 5: $55,1955$.

11 ERLTCH, E.: Med. Clin. N. Amer. 47, 1963.

12 FINNERTY, F. A., Y BUCHOLZ, J.A.M.A. 166: 141, 1958.

13 FARREL, G. L., RAUSCHKOLB, E. W., FLEMING, R. B., YATSU, F. M.: 33th Meet Endocrine Soc. N.Y., Mayo, 1957.

14 FLOWERS, CH. E., GRIZZLE, J. E. EASTERLING, W. E., BLANCHARD, D.: Am. J. Obst. and Gynec. 84: 919, 1962.

15 GUYTON, A. C.: "Tratado de Fisiología Médica". 3a. Ed. 1011, 1969.

16 JONES, K., JONES, A., RINDEL, J., TAll, R., BULBROOK, F., y GREENWOOD: Acta Endocrinol. 30: 321, 1959.

17 KUMAR, D., y BARNES, A.: Obstet. Gynec. Survey 15: 625, 1960.

18 KUMAR, D., FELTHAM, L. A., GORNALL, A. G.: Lancet 1: 541, 1969.

19 LAROCHE, C. L., y COQUET, R.: Encyclopedie Med. Chirurg. Therapetique. 111, 25, 356-10A, 1964.

20 LEUTSCHER, J. A., y CURTIS, R. H.: Ann. Intern. Med. 43: 43, 1958.

21 MAGLIAVACCA, A., y BOMPIANI, A.: Gynecol. Obstet. (Paris) 60: 443, 1961.

22 MAROUDIS, D., HALKINDAKIS, J., PSILAKIS, D., y SAKLARIDES, J.: J. Inst. Coll. Surg. 42: $175,1964$. 
23 MASSEY, F.: Pennsylvania Med. J. 66: 40, 1963.

24 MARTIN, J. D., y MILLS, J. H.: Brit. Med. J. 2 : $571,1956$.

25 PATTERSON, W. B.: "Hiperplasia suprarrenal fetal y su relación con la Toxemia". Tongg Publ. Co. Hawaii. Honolulu, 1973.

26 PRUNTY, F. T. G.: "Modern Trends in Endocrinology". H. Gardiner-Hill London.

Butterwort \& Co.

27 RAUSCHOKOLB, E. W., y FARREL, G.: Endocrinol. 59: 526, 1956.

28 RICHTER, R., YA RNOLD, M.: Gynaecologia. 152: 248,1961 .
29 RINSLER, M. G., y RIGBY, B.: Brit. Med. J. 2 : $966,1957$.

30 ROBINSON, M.: Lancet. 1. 178, 1962.

31 RODRIGUEZ ARGUELLES, J.: "La Toxemia del Embarazo". Talleres Gráficos La Nación. México, 1964.

32 SANCHEZ, F., GOMEZ, J. A., MARTINEZ, C., y SILVA, C.: III Cong. Uruguayo de Toc. Gin. 111, 95, Memoria, 1960.

33 WILSON, G. M.: Diuretics. Brit. Med. J. A. : $285,1963$.

34 ZUSPAN, F. P.: Cls. Obst. y Ginec. 954, 1966.

J. A. GOMEZ PALACINO - Tr. 1a. No 83-33 - Bogotá, Colombia S. A. 